\section{Validation of specimen pooling using the Cepheid Xpert Xpress SARS-CoV-2 assay}

Sir,

Severe acute respiratory syndrome coronavirus 2 (SARSCoV-2) disease (COVID-19) is the latest respiratory tract viral disease to be declared a global pandemic. Throughout the pandemic, laboratory diagnosis of SARS-CoV-2 infection in individual patients has underpinned contact tracing efforts performed by Australian government health departments. The current gold standard for the laboratory detection of SARS-CoV-2 infection (e.g., nasal and/or throat swab) is real-time reverse-transcriptase polymerase chain reaction (RT-PCR) for SARS-CoV-2 nucleic acid. ${ }^{2}$ One of the most widely adopted commercial tests is the Cepheid Xpert Xpress SARS-CoV-2 test ${ }^{3}$ (Cepheid, USA), a rapid automated molecular test that targets two SARS-CoV-2 coding regions, genes E (envelope) and N2 (nucleocapsid). The claimed limit of detection (LOD) is 250 copies $/ \mathrm{mL}$, however, the analytical sensitivity of this assay may be higher with a limit of detection $\leq 100$ copies $/ \mathrm{mL}$.

The overwhelming demand for SARS-CoV-2 testing has placed significant pressure on laboratory resources globally, including the economic burden associated with mass testing but also physical limits in the availability of reagents for nucleic acid extraction and RT-PCR testing. Alongside this is the recognition that, particularly in jurisdictions where the SARS-CoV-2 prevalence has been low, the majority of samples tested from individuals are negative. For example, in a 2week period in Australia between 28 September 2020 and 11 October 2020, 461,888 PCR tests were conducted to diagnose COVID-19 with a positivity rate of $0.05 \%$ reported. ${ }^{4}$ To maintain cost effective levels of testing required for diagnosis and contract tracing, laboratories around the world have explored the efficacy of sample pooling during peak demand, whereby pools of patient samples (of variable size) are initially processed and tested together, and the individual samples present in positive pools are subsequently retested. A number of recent studies have reported that sample pooling strategies are an effective and economical approach to SARS-CoV-2 screening. ${ }^{5-8}$ As expected, sensitivity was lowest in larger pools of samples or in samples with a higher cycle threshold. A comparison of pooling strategies involving sample grouping before or after RNA extraction found that pooling of samples before RNA extraction is preferred for SARS-CoV-2 testing.

In our diagnostic microbiology laboratory, the GeneXpert Xpress SARS-CoV-2 assay has been used to support rapid testing of patients presenting to the hospital's emergency department and other hospital departments during periods of high demand and restricted supply of reagents. To support its use in this setting, in the current study, we performed a fit for purpose validation of specimen pooling using the Xpert Xpress SARS-CoV-2 assay. Sample pooling protocols using different pool sizes were evaluated for use in combination with a diagnostic panel.

To determine the LOD of the Xpert Xpress SARS-CoV-2 assay, a 10-fold dilution series of a high viral load clinical specimen was prepared by dilution using unopened viral transport medium (Tréidlia Biovet, Australia). The LOD dilution series were tested in duplicate to assess reproducibility using the Xpert Xpress SARS-CoV-2 assay on the GeneXpert Dx system (Cepheid, USA). Actual viral copy number per $\mathrm{mL}$ of the diluted samples was assessed using an in-house assay targeting SARS-CoV-2 $\mathrm{N}$ and orflab genes and a standard quantification curve at the NSWHP, Institute of Clinical Pathology and Medical Research (ICPMR) Westmead Laboratory, to assist with determining the LOD of the Xpert Xpress SARS-CoV-2 assay.

To evaluate the impact of sampling pooling, confirmed SARS-CoV-2 negative specimens were used as diluent to simulate specimen pooling of clinical specimens with specimens from the 10 -fold dilution series in the range of $10^{-4}$ to $10^{-9}$. Specimen pooling ratios of 4,6 and 8 specimens were then assessed. Practically, prior to loading the Xpert Xpress SARS-CoV-2 cartridge, $100 \mu \mathrm{L}$ of each specimen was added to a $2 \mathrm{~mL}$ microcentrifuge tube to represent each pooling ratio and then mixed by inversion five times; $300 \mu \mathrm{L}$ of the pooled specimen was then loaded into the cartridge using the sterile pipet provided. Cycle threshold values for the SARS-CoV-2 $\mathrm{E}$ and $\mathrm{N} 2$ genes were then compared against the results obtained from testing neat specimen in order to verify the utility and performance of each specimen pooling ratio. To simulate the 'real-world' impact of sample pooling, an additional set of patient specimens $(n=124)$ were tested neat and also in a 4:1 dilution pool to further validate the latter sample pooling ratio.

With the exception of $10^{-8}$ and $10^{-9}$, the presence of SARS-CoV-2 could be detected at other dilutions for both genes up to $10^{-7}(\mathrm{Ct} \sim 37-40)$, equivalent to 2 SARS-CoV-2 copies/mL (data not shown). These dilutions represented the assay's limit of detection with viral loads significantly higher than those detected in individuals considered infectious with SARS-CoV-2.

Sample pooling revealed that at a $4: 1$ dilution, the $\mathrm{Ct}$ values varied from between 1.1 to 3.4 across the different dilutions and genes tested with an average $\mathrm{Ct}$ variation of 1.9-2.7 for SARS-CoV-2 E and N2 gene assays (compared to the neat specimen), respectively (Table 1 ). For pools of six samples, the $\mathrm{Ct}$ values varied from 1.3 to 4.0 when compared with the neat specimen across the dilutions and target genes with an average change in the Ct of 2.6 and 2.7 for SARSCoV-2 E and N2 genes. Pools of eight samples showed the largest variation in $\mathrm{Ct}$ value from the neat specimen with $\mathrm{Ct}$ values ranging between 2.8 to 8.2 and an average variation in the $\mathrm{Ct}$ of 3.3 and 3.5 for SARS-CoV-2 E and N2 genes. To further validate a 4:1 sample dilution ratio, we tested an additional 124 patient specimens prospectively. Five neat specimens were found to be positive (Ct range 12.7-22.9). All of these specimens were also found positive in 4:1 diluted pool with an average change in the $\mathrm{Ct}$ of 1.4 and 1.5 for SARS-CoV-2 E and N2 genes, respectively (data not shown).

The results of these fit for purpose experiments suggest that sample pooling is a viable option to allow for higher throughput/reagent savings in medium to low SARS-CoV-2 prevalence settings. Based on our comparison of different pool sizes, the optimal selected protocol involved the pooling 
Table 1 Results of GeneXpert Xpress SARS-CoV-2 assay pooling of clinical specimens

\begin{tabular}{lcc}
\hline Pooling protocol & $\begin{array}{c}\text { Average } \Delta \text { pool Ct } \\
\text { E gene }\end{array}$ & $\begin{array}{c}\text { Average } \Delta \text { pool Ct } \\
\text { N2 gene }\end{array}$ \\
\hline 4 to 1 & 1.9 & 2.7 \\
6 to 1 & 2.6 & 2.7 \\
8 to 1 & 3.3 & 3.5 \\
\hline
\end{tabular}

of four samples, where only one sample may be positive and, in effect, diluting the positive specimen 1:4. This result is consistent with the results of similar experiments described by Chong et al. $^{8}$ with an increase in $\mathrm{Ct}$ values following sample pooling, consistent with our own study despite the authors using a different SARS-CoV-2 assay. These authors also concluded that pools of four samples are the optimal pool size, trading assay sensitivity for a notable increase in the efficiency of sample testing. The latter study further concluded that this pooling strategy would be ideal for testing large numbers of samples with very low test-probability, even when there were no concerns over reagent access. ${ }^{8}$ Based on these results, we successfully introduced this sample pooling strategy as a component of our local diagnostic microbiology surge response to outbreaks of SARS-CoV-2 in Western Sydney between June and October 2021, reserving pooled specimens for lower risk patients. In the USA, specimen pooling has been approved as a strategy to increase screening testing capacity for SARS-CoV-2 assays approved for use by the FDA. ${ }^{0}$ Pooling of specimens on the GeneXpert platform is not currently endorsed by the manufacturer and laboratories are required to accredit this variation with NATA as inhouse in vitro diagnostic device (IVD). Of note, NSWHP laboratories are NATA accredited for pooling on several other SARS-CoV-2 NAT assays. The work described in this study, involving pragmatic changes to diagnostic procedures to conserve critical diagnostic reagents, highlights the importance of flexibility in managing a laboratory response to the challenges presented by COVID-19 and other new and emerging infectious disease threats now and into the future.

Conflicts of interest and sources of funding: The authors state that there are no conflicts of interest to disclose.

Rifky Balgahom ${ }^{1}$, Vishal Ahuja ${ }^{2}$, Catherine Pitman ${ }^{2}$, Adam Polkinghorne ${ }^{1,3}$, James Branley ${ }^{1,3}$

${ }^{1}$ Department of Microbiology and Infectious Diseases, New South Wales Health Pathology-Nepean, Penrith, NSW, Australia; ${ }^{2}$ New South Wales Health Pathology - Public Health Pathology State-wide Service, Westmead, NSW, Australia; ${ }^{3}$ Nepean Clinical School, Faculty of Medicine and Health, University of Sydney, Kingswood, NSW, Australia

Contact Dr James Branley.

E-mail: james.branley@health.nsw.gov.au

1. Jin Y, Yang H, Ji W, et al. Virology, epidemiology, pathogenesis, and control of COVID-19. Viruses 2020; 12: 372

2. D'Cruz RJ, Currier AW, Sampson VB. Laboratory testing methods for novel severe acute respiratory syndrome-coronavirus-2 (SARS-CoV-2). Front Cell Dev Biol 2020; 8: 468.
3. Loeffelholz MJ, Alland D, Butler-Wu SM, et al. Multicenter evaluation of the Cepheid Xpert xpress SARS-CoV-2 test. J Clin Microbiol 2020; 58: e00926-20.

4. COVID-19 National Incident Room Surveillance Team. COVID-19 Australia: epidemiology report 27. Fortnightly reporting period ending 11 October 2020. Commun Dis Intelligence 2020; 44: 1-20.

5. Mutesa L, Ndishimye P, Butera Y, et al. A pooled testing strategy for identifying SARS-CoV-2 at low prevalence. Nature 2020; 589: 27680 .

6. Volpato F, Lima-Morales D, Wink PL, et al. Pooling of samples to optimize SARS-CoV-2 diagnosis by RT-qPCR: comparative analysis of two protocols. Eur J Clin Microbiol Infect Dis 2020; 40: 889-92.

7. Cabrera Alvargonzalez JJ, Rey Cao S, Perez Castro S, et al. Pooling for SARS-CoV-2 control in care institutions. BMC Infect Dis 2020; 20: 745.

8. Chong BSW, Tran T, Druce J, Ballard SA, Simpson JA, Catton M. Sample pooling is a viable strategy for SARS-CoV-2 detection in lowprevalence settings. Pathology 2020; 52: 796-800.

9. Sparks R, Balgahom R, Janto C, Polkinghorne A, Branley J, Samarasekara H. An Australian diagnostic microbiology surge response to the COVID-19 pandemic. Diagn Microbiol Infect Dis 2021; 100: 115309

10. Centers for Disease Control and Prevention. Interim Guidance for Use of Pooling Procedures in SARS-CoV-2 Diagnostic, Screening, and Surveillance. Centers for Disease Control and Prevention, 2020.

DOI: https://doi.org/10.1016/j.pathol.2021.12.286

\section{Prolonged granulocyte transfusions sourced from buffy coats used to treat Aspergillus spp. infection in chronic granulomatous disease}

Sir,

Chronic granulomatous disease (CGD) is caused by inherited defects of the phagolysosomal NADPH-oxidase, leading to impaired neutrophil function and a high lifetime risk of invasive mould infection. We describe the successful outcome of an adult CGD patient with disseminated Aspergillus fischeri, with suspected triazole-resistance and clinical and radiological progression despite multiple high dose antifungals. His invasive fungal disease (IFD) was subsequently controlled by administering a prolonged course of granulocyte transfusions (GT) sourced from buffy coats (BCs).

A 37-year-old male with CGD (autosomal recessive $\mathrm{p} 47^{\text {phox }}$ deficiency) underwent donor-after-brain-death renal transplantation. Pre-transplantation, he had no active infections (including no evidence of latent mould infection on chest X-ray), and was receiving prophylactic itraconazole $200 \mathrm{mg}$ daily (Sporanox) and trimethoprim-sulfamethoxazole $160 / 800 \mathrm{mg}$ daily with therapeutic drug monitoring of itraconazole achieving serum levels between 100 and $300 \mu \mathrm{g} / \mathrm{L}$. The donor had no risk factors for mould infection. Immune suppression consisted of intravenous (IV) basiliximab and methylprednisolone perioperatively, followed by maintenance tacrolimus, mycophenolate mofetil and prednisolone as per standard institutional protocols.

His transplant was complicated by biopsy-confirmed graft rejection at 2 weeks, requiring pulse high-dose methylprednisolone followed by a prolonged weaning dose of oral prednisone. At 12 weeks he developed headaches, vomiting and visual disturbance. Brain magnetic resonance imaging revealed ring enhancing lesions in the left parietal and right occipital lobes suspicious for IFD (Fig. 1), requiring a 